\title{
MUDANÇAS NAS FUNÇÕES DO PROFISSIONAL DE CONTABILIDADE APÓS A IMPLEMENTAÇÃO DO ERP
}

\section{CHANGES IN ACCOUNTANT ROLES AFTER AN ERP IMPLEMENTATION}

\section{Maria do Céu Gaspar Alves}

Investigadora do NECE/Universidade da Beira Interior (UBI). Doutora em Gestão com Especialização em Contabilidade pela UBI. Professora Auxiliar no Departamento de Gestão e Economia da UBI. E-mail: mceu@ubi.pt (Portugal)

\section{Sergio Ivo Amaral Matos}

Mestre em Contabilidade pela Universidade do Minho (UMinho). E-mail: serg.ivomatos@gmail.com (Portugal) 


\title{
MUDANÇAS NAS FUNÇÕES DO PROFISSIONAL DE CONTABILIDADE APÓS A IMPLEMENTAÇÃO DO ERP
}

\section{RESUMO}

Os sistemas ERP têm merecido alguma atenção dos investigadores, sendo a maioria dos estudos efetuados na fase de implementação do ERP. Porém, é na fase seguinte, fase pósimplementação, que se esperam maiores mudanças na contabilidade e nas competências exigidas ao profissional de contabilidade. Assim, e pretendendo colmatar esta lacuna, esta investigação incide nas competências exigidas ao profissional de contabilidade na fase pósimplementação do ERP. Para isso, é efetuado um estudo empírico, por meio de um inquérito por questionário, tendo se alcançado uma taxa de resposta de $51 \%$. Os resultados obtidos indicam, por um lado, que o profissional de contabilidade tem de desenvolver novas competências para responder aos novos desafios colocados com a implementação do ERP. Por outro lado, que ele passa a necessitar de menos tempo para desempenhar as tarefas tradicionais, podendo dedicar-se a novas tarefas na organização. Ou seja, verifica-se uma mudança na gestão do tempo e na natureza das tarefas exigidas ao profissional de contabilidade.

Palavras-chave: Contabilidade de Gestão. Sistemas ERP. Mudanças na Contabilidade de Gestão. Profissional de contabilidade.

\section{CHANGES IN ACCOUNTANT ROLES AFTER AN ERP IMPLEMENTATION}

\begin{abstract}
Recently ERP systems have received some attention from researchers and most studies are carried out on the implementation phase. However, major changes in the accounting field and in the skills required to accountant are expected in the post-implementation phase. In order to bridge this gap, this research focuses on the skills required to accountant in the ERP postimplementation phase. A survey research is performed, having achieved a response rate of $51 \%$. The results indicate that the ERP implementation requires new skills and a change in the management accountant tasks.
\end{abstract}

Keywords: Management accounting. ERP Systems. Changes in management accounting. Accountant. 


\section{INTRODUÇÃO}

A complexidade crescente das organizações que tem caraterizado os últimos tempos, advém de fenómenos como o desenvolvimento tecnológico e a globalização dos negócios (Alves \& Matos, 2013). Esta crescente complexidade faz com que sejam diariamente, produzidas, tratadas e armazenadas grandes quantidades de informação em sistemas de informação integrados, nomeadamente em sistemas ERP- Entreprise Resource Planning Systems (Shang \& Seddon, 2002). Este sistema permite às organizações integrarem todos os seus processos de negócio, permite uma maior rapidez ao nível do processamento de transações, do reporting e da análise de informações, e também permite obter informação em tempo real (Davenport, 1998; Fahy \& Linch, 1999; Granlund \& Malmi, 2002; Hyvönen, 2003; Scapens \& Jazayeri, 2003). Consequentemente, esse sistema aumenta a flexibilidade na geração de informação, o que permite aos gestores responderem de forma mais eficiente às mudanças que ocorrem no ambiente empresarial (Spathis \& Constantinides, 2004). Atualmente, os sistemas ERP, as bases de dados, a internet e a intranet representam novas formas de organização das operações que permitem integrar algumas atividades e gerir as organizações em tempo real (Berry, Coad, Harris, Otley \& Stringer, 2009).

São vários os investigadores, que estudaram o impacto do sistema ERP na contabilidade de gestão (Fahy \& Lynch, 1999; Granlund \& Malmi, 2002; Scapens \& Jazayeri, 2003) e, embora alguns autores considerem que a implementação do sistema não provoca mudanças significativas na contabilidade de gestão (Hyvönen, 2003; Spathis \& Constantinides, 2004), outros sugerem que as características do ERP podem ser um indutor na adoção de modernas técnicas de contabilidade de gestão (Booth, Matolcsy \& Wieder, 2000). Por outro lado, alguns estudos apontam para a existência de um impacto direto nas características do trabalho do profissional de contabilidade de gestão (Caglio, 2003; Scapens \& Jazayeri, 2003). Assim, constata-se, que o sistema ERP reduz o tempo necessário para a execução das tarefas de rotina e, assim, o profissional de contabilidade passa a dispor de mais tempo para realizar análises da informação (Granlund \& Malmi, 2002). De tal modo, que para Scapens e Jazayeri (2003), a implementação do sistema ERP pode provocar uma diminuição da necessidade de profissionais de contabilidade de gestão, pois os gestores de linha teriam a possibilidade de gerir seus próprios custos e orçamentos. Nesse contexto, o profissional de contabilidade passaria a desempenhar funções de consultor interno ou conselheiro de gestão (Lambert \& Sponem, 2012). Assim sendo, constata-se que nesse novo ambiente, o papel do contabilista de gestão está a mudar (Granlund \& Malmi, 2002; Caglio, 2003; Lodh \& Gaffiki, 
2003; Scapens \& Jazayeri, 2003; Rom \& Rohde, 2006), e esta mudança pressupõe a aquisição de novas competências, sobretudo na área das tecnologias de informação (Spathis \& Constantinides, 2004). Mas existe ainda um fenómeno de "hibridização" das funções do contabilista em organizações que implementaram o sistema ERP (Caglio, 2003; Lambert \& Sponem, 2012), isto é, observa-se que o conhecimento contabilístico é difundido por toda a organização, chegando mesmo a pessoas que não têm formação em contabilidade.

A clara alteração do ambiente de trabalho do profissional de contabilidade e a fragmentação da investigação já realizada reforçam a necessidade de novos estudos. A presente investigação procura dar um contributo nesta matéria.

Por outro lado, constata-se que a maioria dos estudos se tem centrado na fase de implementação do sistema ERP (Fahy \& Lynch, 1999; Booth et al., 2000; Granlund \& Malmi, 2002; Scapens \& Jazayeri, 2003; Spathis \& Constantinides, 2004; Spathis, 2006), tendo a fase posterior à implementação recebido pouca atenção por parte da comunidade científica (Aernoudts, Boom, Vosselman \& Pijl, 2005). Assim, e embora a literatura faça referência a vários estudos que apenas encontraram um fraco ou moderado impacto do sistema ERP na contabilidade de gestão (Booth et al., 2000; Granlund \& Malmi, 2002; Scapens \& Jazayeri, 2003), existem autores (Spathis \& Constantinides, 2004; Spathis, 2006) que argumentam que as mudanças na contabilidade de gestão não são imediatas. Assim, é à medida que o tempo for decorrendo, após a implementação do ERP, que se devem esperar mudanças na contabilidade de gestão. Esses autores acrescentam que essas mudanças não aparecem espelhadas nos estudos realizados, porque a maioria das organizações estudadas possuem um sistema ERP implementado há muito pouco tempo. Constata-se assim que existe a necessidade de se desenvolver mais investigação focada na fase pós-implementação do ERP (Abdinnour \& Saeed, 2015; Grabski, Leech \& Schmidt, 2011; Ruivo, Oliveira \& Neto, 2014). Procurando colmatar esta lacuna, este trabalho irá incidir sobre organizações que já implementaram o sistema ERP há algum tempo. Acresce ainda, a necessidade da realização de estudos em empresas portuguesas, pois até ao momento são muito poucos os estudos do género realizados em Portugal (Ruivo et al., 2014). De facto, a forte carência de investigação na área da contabilidade de gestão em Portugal contribuiu significativamente para a relevância e oportunidade deste estudo. Em termos práticos, este estudo procura contribuir para um maior conhecimento da realidade portuguesa. Assim, uma das principais metas desta pesquisa consiste na recolha de evidências que permitam aprofundar o conhecimento da realidade portuguesa, sobre os efeitos do sistema ERP nas funções e no papel do profissional de contabilidade em organizações que implementaram com sucesso este sistema. E mais 
concretamente, nas capacidades/competências requeridas ao contabilista de gestão e no tempo necessário para realizar suas tarefas.

Para isso, colocaram-se as seguintes questões: A implementação do sistema ERP provoca mudanças no trabalho do contabilista de gestão? Que competências são exigidas a estes profissionais? Terá este profissional tempo disponível para analisar a informação produzida? Para responder a estas questões é efetuada, em seguida, uma revisão da literatura, a partir da qual são formuladas hipóteses de investigação posteriormente testadas num estudo empírico.

\section{REVISÃO DA LITERATURA, HIPÓTESES E MODELO DE INVESTIGAÇÃO}

No início dos anos de 1980, o profissional de contabilidade desempenhava um cargo de assessoria na organização, encontrava-se afastado do processo produtivo, tendo muito pouco contacto com os gestores operacionais. Não participava no processo de tomada de decisão e funcionava como assessor dos decisores, sendo frequentemente informado das decisões depois de elas terem sido tomadas. A maior parte do seu tempo era despendida com os aspetos técnicos da contabilidade. Com o decorrer dos anos, os avanços tecnológicos libertaram-no dos aspetos mecânicos da contabilidade, o contabilista de gestão passa hoje menos tempo a elaborar relatórios padronizados e mais tempo a analisar e interpretar informação. Trabalha em equipa, desenvolve uma extensa comunicação com os outros membros da organização e está ativamente envolvido no processo de tomada de decisão (Carnegie \& Napier, 2010).

Em paralelo, os avanços tecnológicos, conduziram as organizações à implementação de sistemas de informação mais desenvolvidos, como é o caso dos sistemas ERP. Estando a contabilidade de gestão e o profissional de contabilidade no centro da produção de informações para a tomada de decisão, e sendo o sistema ERP uma poderosa ferramenta para o seu processamento e produção, os efeitos potenciais de tais sistemas sobre a contabilidade de gestão e sobre o profissional de contabilidade tornam-se evidentes. Nesse contexto, são vários os autores que consideram que o papel do contabilista de gestão se modificou com a implementação do sistema ERP (Granlund \& Malmi, 2002; Newman \& Westrup, 2005; Ribeiro \& Oliveira, 2009; Scapens \& Jazayeri, 2003; Spathis \& Constantinides, 2004).

À primeira vista o sistema ERP vem reduzir as necessidades de pessoal nas áreas da contabilidade, nomeadamente nas atividades de recolha e processamento de informação; mas, ao mesmo tempo, coloca novas exigências e abre novas oportunidades aos profissionais de 
contabilidade (Newman \& Westrup, 2005). Os trabalhos de Scapens, Jazayeri e Scapens (1998) reforçam essas ideias, ao estudarem organizações que implementaram sistemas ERP, os autores constatam que em alguns casos o acesso à informação, por parte dos utilizadores, passou a ser direto. Assim, tendem a extinguirem-se algumas atividades típicas dos departamentos de contabilidade. Em contrapartida, ganham relevância as funções de apoio e interpretação da informação, atividades para as quais os profissionais de contabilidade passam a ter mais tempo disponível. Neste contexto, exige-se, pois, aos profissionais da contabilidade uma compreensão dos processos de negócio da organização. Assim, o contabilista de gestão está agora a desempenhar funções mais direcionadas para a gestão (Granlund \& Malmi, 2002; Quattrone \& Hopper, 2001; Scapens \& Jazayeri, 2003). Competências multidisciplinares são exigidas deles (Lodh \& Gaffikin, 2003). Scapens e Jazayeri (2003) consideram mesmo que os trabalhos rotineiros foram eliminados e que os contabilistas de gestão desempenham um papel mais abrangente. Dessa forma, parece haver consenso sobre o facto de os contabilistas se envolverem na gestão em geral e atuarem como consultores de gestão. Surge assim um novo perfil profissional, com o profissional de contabilidade de gestão a transitar de um papel de técnico de contabilidade (bean-counters) para uma função de analista de negócios (Burns \& Baldvinsdottir, 2005; Carnegie \& Napier, 2010) ou de parceiro de negócios (business partner) (Lambert \& Sponem, 2012; Simons, 2012).

Macarrone (2000), alega que os sistemas ERP colocam novos desafios aos contabilistas de gestão. Assim, um crescente nível de competências em inovação tecnológica ser-lhes-á solicitado para dominar as novas situações criadas com a implementação do sistema ERP. Por outro lado, os contabilistas de gestão estão a conquistar um novo papel com os sistemas integrados de informação. A introdução dos sistemas ERP criou novas posições, "híbridas" para o contabilista de gestão (Caglio, 2003). Eles cumprem agora tarefas de consultores de gestão e tarefas relacionadas com a manutenção dos sistemas integrados de informação em adição às tarefas de contabilidade de gestão. Além disso, é notório que a proatividade dos contabilistas é importante, se quiserem valorizar o seu próprio papel. Contudo, o profissional de contabilidade deverá estar consciente que controlar os sistemas integrados de informação acarreta grandes pressões, pois muitas pessoas dependem do seu bom funcionamento (Caglio, 2003). Se o contabilista de gestão não estiver à altura do desafio, irá perder a oportunidade que outros grupos profissionais, tais como os profissionais de sistemas de informação, estão interessados em aproveitar (Newman \& Westrup, 2005).

Podemos ainda referir, que com o ERP a contabilidade de gestão fica descentralizada, ou seja, num nível mais operacional ela é conduzida por não contabilistas. O sistema ERP tem 
muitas rotinas incluídas que, por exemplo, atualizam automaticamente os registos quando são inseridos dados noutras partes do sistema. O trabalhador introduz os dados operacionais no sistema e este encarrega-se de fazer o processamento contabilístico habitual (Scapens \& Jazayeri, 2003). A contabilidade de gestão tornou-se assim numa atividade descentralizada. Por outro lado, o sistema integrado de informação, capaz de distribuir informações por toda a empresa, fornece aos gestores de linha conhecimentos de contabilidade, para que estes conheçam e controlem seus próprios gastos e orçamentos (Caglio, 2003; Scapens \& Jazayeri, 2003). Dessa forma, também o controlo passa a estar descentralizado. Quattrone e Hopper (2001, 2005) vão mais longe argumentando que nessas organizações todas as pessoas podem optar por exercer algum tipo de controlo.

Tais mudanças assustam alguns profissionais de contabilidade os quais temem que o acesso fácil aos dados por meio do sistema ERP possa reduzir a importância da informação produzida por eles se houver uma inadequada comunicação entre os fornecedores destas soluções / tecnologias (IT-experts) e os utilizadores finais (gestores) (Maccarrone, 2000). Segundo o autor, o medo da "perda de poder e controlo", a médio e longo prazo (devido à facilidade de acesso à informação disponibilizada pelo sistema ERP), está presente nos contabilistas de gestão.

É de notar que a alteração do papel do profissional de contabilidade é um tema recorrente nos estudos anteriores e transversal a diversos posicionamentos metodológicos (Caglio, 2003; Scapens \& Jazayeri, 2003). Relativamente unânime é a eliminação de tarefas rotineiras de recolha e processamento de informação permitida pelos sistemas ERPs. Mas, coexistem duas visões na literatura quanto à "hibridização" do papel daqueles profissionais. Alguns autores salientam o alargamento do papel do profissional de contabilidade no sentido de um maior envolvimento no processo de gestão e na produção de informação mais prospetiva (Burns \& Baldvinsdottir, 2005). Outros, focam a descentralização de funções e a difusão de formas de controlo, o que poderá representar um risco e conduzir à marginalização dos profissionais de contabilidade e controlo de gestão (Dechow \& Mouritsen, 2005).

Os sistemas ERP vêm sendo cada vez mais usados pelas organizações. Essa realidade requer que, na sua implementação, estejam envolvidos profissionais de diversas áreas, inclusive profissionais de contabilidade. As implementações de sistemas ERP ocorrem em muitas ocasiões, com uma série de limitações. Para reduzir estas limitações é importante um grande envolvimento dos intervenientes durante todo o processo de implementação. O conhecimento dos processos e da importância das informações que tem o profissional de contabilidade, pode fazer a diferença entre uma implementação de sucesso e uma 
implementação problemática (Vanti, Ferreira, Janikian \& Ferro, 2003). Embora existam alguns riscos técnicos na implementação dos sistemas ERP, vários estudos têm demonstrado que o maior risco se situa ao nível da competência dos recursos humanos (Avital \& Vandenbosch, 2000; Wu, Shin \& Heng, 2007), entre eles do profissional de contabilidade.

Para o desempenho das novas funções do profissional de contabilidade, resultantes da implementação do sistema ERP, são necessárias novas competências como a capacidade de comunicação interpessoal e um conhecimento mais alargado das matérias de gestão (Newman \& Westrup, 2005; Scapens \& Jazayeri, 2003). Dessa forma, são exigidos novos conhecimentos, nomeadamente a habilidade de saber comunicar e trabalhar em equipa, de possuir sólidos conhecimentos analíticos de contabilidade e um conhecimento profundo do funcionamento do negócio (Alves, 2003). Em paralelo, exigem-se bons conhecimentos em tecnologias de informação (Spathis \& Constantinides, 2004).

O contabilista de gestão é um elemento essencial no processo de implementação do sistema ERP, os seus conhecimentos e a sua visão dos negócios e atividades da empresa contribuem para uma implementação bem-sucedida. Contudo, é indispensável que a atuação do profissional de contabilidade seja suportada por uma formação académica sólida e atualizada.

Dadas as questões de investigação apresentadas na introdução, e à luz da revisão da literatura efetuada são formuladas duas hipóteses de investigação.

Hipótese 1 - As capacidades/competências requeridas ao contabilista de gestão aumentaram após a implementação do sistema ERP.

Hipótese 2 - O tempo necessário para o contabilista de gestão realizar suas tarefas mudou após a implementação do sistema ERP.

Para operacionalizar o estudo e testar as hipóteses, foi definido um modelo de investigação (Figura 1) composto por duas variáveis para determinar o impacto do sistema ERP no papel e nas tarefas do contabilista de gestão. 
Figura 1 - Modelo de investigação

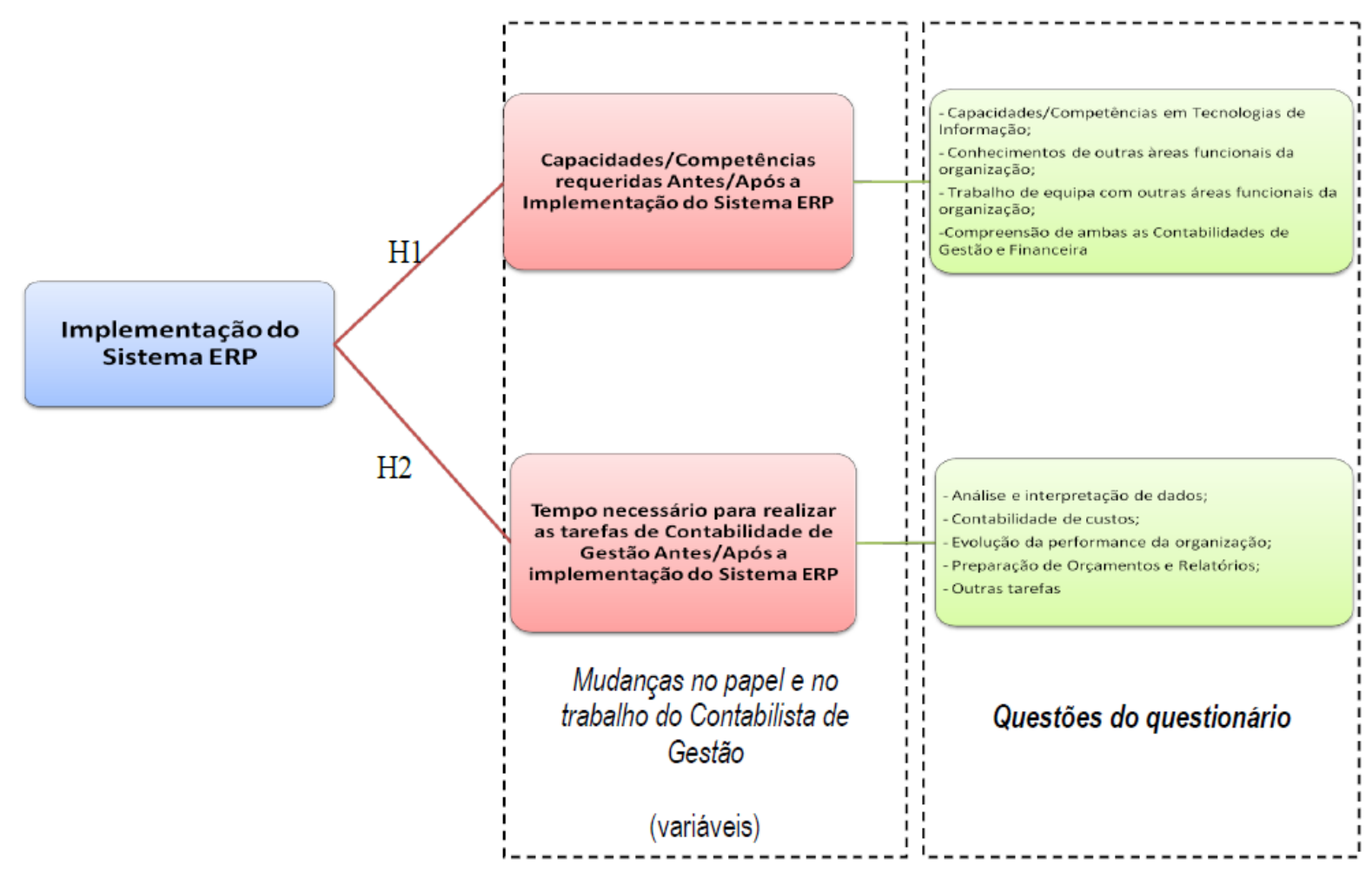

Fonte: Elaborada pelos autores (2017).

Com a primeira variável, pretende-se averiguar se o sistema ERP requer novas capacidades/competências, aos profissionais de contabilidade. Para isso, é solicitado aos inquiridos que avaliem as capacidades/competências, consideradas importantes pela literatura. Ou seja, capacidades/competências em tecnologias de informação (Macarrone, 2000; Spathis \& Constantinides, 2004; Spathis, 2006); conhecimentos de outras áreas funcionais da organização (Fahy \& Linch, 1999); trabalho de equipa com outras áreas funcionais da organização (Sayed, 2006); e compreensão de ambas as contabilidades, de gestão e financeira (Caglio, 2003). Serão comparadas as respostas dadas pelos inquiridos sobre as capacidades/competências requeridas antes e após a implementação do sistema ERP.

Com a segunda variável procura-se estudar o tempo necessário para realizar as tarefas de contabilidade de gestão. Aos inquiridos é solicitada uma estimativa do tempo despendido (em percentagem de tempo de trabalho global), no desempenho de determinadas tarefas antes e após a implementação do sistema ERP. As tarefas analisadas são: a análise e interpretação de dados (Granlund \& Malmi, 2002); a contabilidade de custos (Granlund \& Malmi, 2002); a evolução da performance da organização (Scapens \& Jazayeri, 2003); a preparação de orçamentos e relatórios (Fahy \& Linch, 1999) entre outras tarefas. 


\section{METODOLOGIA DO ESTUDO EMPÍRICO}

Depois de definidos os objetivos e formuladas as hipóteses a testar, é necessário definir a população-alvo do inquérito, que neste estudo, é constituída por 130 organizações que implementaram com sucesso o sistema ERP da SAP. Para a definição dessa população foi utilizada a listagem de empresas divulgada pela SAP no seu site da internet (www.sap.pt). Como informador-chave foi escolhido o profissional de contabilidade.

Em termos metodológicos optou-se por um estudo empírico quantitativo, uma vez que o paradigma de investigação subjacente ao trabalho desenvolvido é positivo. Os dados utilizados nesta investigação foram recolhidos através de um inquérito por questionário. Este foi implementado por meio de uma aplicação disponibilizada no site www.smartsurveyor.com. A técnica utilizada neste estudo consistiu numa combinação da internet com um questionário remetido via e-mail (Ganassali, 2008). Segundo Ferreira e Sarmento (2009), o recurso à internet permite obter elevada taxa de respostas num curto período de tempo e pode, em certas situações, ser um meio com custo baixo.

O questionário antes de ser aplicado, foi revisto, testado e validado, junto de um grupo de pessoas do meio académico e do maior fornecedor de sistemas ERP em nível mundial que é a SAP, neste caso a SAP Portugal. Depois de ligeiros ajustamentos o questionário foi lançado. No total obtiveram-se 66 respostas e foram considerados 65 questionários válidos. Assim, a taxa de resposta foi de 50,77\% (66 respostas ao questionário em 130). Finalmente, os dados recolhidos foram tratados e analisados com a ajuda do programa Statistical Package for Social Sciences (SPSS) for Windows.

\section{RESULTADOS DE ESTUDO EMPÍRICO}

Na revisão da literatura efetuada, as conclusões de alguns trabalhos que alegam não existir impacto da implementação do ERP na contabilidade são contestadas pelo facto de esses estudos terem sido realizados logo após a implementação do sistema ERP, ou seja, muito cedo para que se possa avaliar o real impacto da implementação do sistema ERP. Tendo em conta tal situação, foram colocadas no questionário duas perguntas: "Há quantos anos utiliza o sistema ERP?" e "Em que ano se iniciou o processo de implementação do ERP?". Os resultados obtidos permitem-nos concluir que neste estudo essa limitação é ultrapassada uma vez que o sistema ERP está implementado, em média, há mais de seis anos 
nas organizações estudadas, e apenas $12 \%$ das organizações implementaram o sistema há menos de três anos (Quadro 1).

Quadro 1 - Tempo de utilização do sistema ERP.

\begin{tabular}{llll}
\hline Variável & $\boldsymbol{M}$ & $\boldsymbol{D P}$ & Limites \\
Anos que utiliza o & 6,29 anos & 3,38 anos & $1-14$ anos \\
Sistema ERP & & \\
\hline laborado pelos autores (2017).
\end{tabular}

Fonte: Elaborado pelos autores (2017).

Destaca-se ainda o facto de a maioria dos inquiridos já exercer seu cargo/função há mais de seis anos - o que significa que muitos acompanharam o processo de implementação do sistema ERP.

Uma das questões de investigação deste trabalho consiste em saber qual a percepção do impacto do sistema ERP no trabalho do profissional de contabilidade de gestão. Desse modo, procurou-se saber se na opinião dos inquiridos, a implementação do sistema ERP tinha alterado as características e a importância do trabalho do profissional de contabilidade. Para isso, foi solicitado aos inquiridos que se pronunciassem sobre os efeitos do sistema ERP em diversos aspectos relacionados com o seu trabalho, referidos no Quadro 2.

Constata-se que o profissional de contabilidade considera que após a implementação do sistema ERP, a importância do seu trabalho aumentou e o trabalho necessário para realizar as suas tarefas diminuiu. Por sua vez, os conhecimentos necessários em tecnologias de informação, o rigor necessário na realização do trabalho, a liberdade/autonomia na forma de trabalhar, a necessidade de conhecimentos das outras áreas funcionais, e a necessidade de trabalhar em equipa com as outras áreas funcionais aumentaram (Quadro 2).

Quadro 2- Efeitos que o sistema ERP teve sobre o trabalho do contabilista de gestão.

\begin{tabular}{|c|c|c|c|c|c|c|c|c|c|c|}
\hline \multirow{2}{*}{$\begin{array}{l}\text { Itens } \\
(N=65)\end{array}$} & \multicolumn{2}{|c|}{ Dimin Muito } & \multicolumn{2}{|c|}{ Dimin. Pouco } & \multicolumn{2}{|c|}{ Não Mudou } & \multicolumn{2}{|c|}{ Aumen. Pouco } & \multicolumn{2}{|c|}{ Aumen. Muito } \\
\hline & $\bar{n}$ & $\%$ & $n$ & $\%$ & $\bar{n}$ & $\%$ & $\bar{n}$ & $\%$ & $n$ & $\%$ \\
\hline Importância do meu trabalho. & - & - & 2 & 3,1 & 28 & 43,1 & 19 & 29,2 & 16 & 24,6 \\
\hline $\begin{array}{l}\text { Trabalho necessário para a } \\
\text { realização das minhas tarefas. }\end{array}$ & 9 & 13,8 & 23 & 35,4 & 11 & 16,9 & 15 & 23,1 & 7 & 10,8 \\
\hline $\begin{array}{l}\text { Conhecimentos necessários em } \\
\text { tecnologias de informação. }\end{array}$ & - & - & - & - & 8 & 12,3 & 43 & 66,2 & 14 & 21,5 \\
\hline $\begin{array}{l}\text { Rigor necessário na realização do } \\
\text { meu trabalho. }\end{array}$ & 1 & 1,5 & - & - & 27 & 41,5 & 19 & 29,2 & 18 & 27,7 \\
\hline $\begin{array}{l}\text { Liberdade/autonomia na forma de } \\
\text { trabalhar. }\end{array}$ & 1 & 1,5 & 10 & 15,4 & 17 & 26,2 & 20 & 30,8 & 17 & 26,2 \\
\hline $\begin{array}{l}\text { Necessidade de conhecimentos das } \\
\text { outras áreas funcionais. }\end{array}$ & - & - & - & - & 13 & 20,0 & 33 & 50,8 & 19 & 29,2 \\
\hline 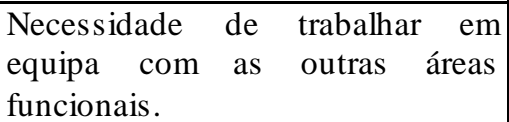 & - & - & - & - & 13 & 20,0 & 31 & 47,7 & 21 & 32,3 \\
\hline
\end{tabular}




\begin{tabular}{|l|l|l|l|l|l|l|l|l|l|l|}
\hline Estabilidade do posto de trabalho. & 1 & 1,5 & 2 & 3,1 & 46 & 70,8 & 12 & 18,5 & 4 & 6,2 \\
\hline $\begin{array}{l}\text { Conhecimentos de contabilidade } \\
\text { financeira e de contabilidade de } \\
\text { gestão. }\end{array}$ & - & - & - & - & 38 & 58,5 & 21 & 32,3 & 6 & 9,2 \\
\hline
\end{tabular}

Fonte: Elaborado pelos autores (2017).

As principais mudanças no papel e no trabalho do contabilista de gestão com a implementação do ERP são avaliadas neste estudo empírico, em termos das capacidades/competências requeridas e do tempo necessário para realizar as tarefas de contabilidade de gestão, antes e após a implementação do sistema ERP.

Em primeiro lugar, são apresentados os resultados da perceção que os profissionais de contabilidade têm das capacidades/competências, que thes são exigidas antes e após a implementação do sistema ERP. Foram analisadas quatro capacidades/competências, consideradas importantes na literatura sobre o tema (Caglio, 2003; Fahy \& Linch, 1999; Macarrone, 2000; Spathis \& Constantinides, 2004; Sayed, 2006; Spathis, 2006).

Os resultados obtidos indicam que após a implementação do ERP aumentou a importância destas quatro capacidades/competências. Antes da implementação do ERP o profissional de contabilidade já considerava importantes: 1) as capacidades/competências em tecnologias de informação $(n=36 ; 55,4 \%) ; 2)$ o conhecimento de outras áreas funcionais da organização $(n=44 ; 67,7 \%) ; 3)$ o trabalho de equipa com outras áreas funcionais da organização $(n=43 ; 66,2 \%)$ e a compreensão de ambas as contabilidades de gestão e financeira $(n=53 ; 81,5 \%)$. Mas, após a implementação do ERP a importância dessas competências aumentou, ou seja, passamos a ter $61(93,8 \%)$ profissionais de contabilidade a considerarem como importante as capacidades/competências em tecnologias de informação, o conhecimento de outras áreas funcionais da organização, o trabalho de equipa com outras áreas funcionais da organização e $62(95,4 \%)$ profissionais de contabilidade a considerarem como importante a compreensão de ambas as contabilidades de gestão e financeira.

Para averiguar o impacto do sistema ERP foi ainda solicitado aos inquiridos que, tendo em consideração o desempenho do seu cargo, avaliassem a importância de um conjunto de capacidades e competências (referidas no Quadro 3), antes e após a implementação do sistema ERP. Para analisar os resultados obtidos, foi aplicado o Teste de Friedman para verificar se as diferenças entre os dois grupos (Antes e Após a implementação do ERP) são estatisticamente significativas, em termos da importância das diferentes capacidades/competências requeridas com o ERP. 
Antes de se aplicar o teste de Friedman, realizou-se o teste de Kolmogorov-Smirnov para aferir sobre a normalidade dos valores da amostra. Após a aplicação do teste de normalidade, este evidenciou não haver normalidade na distribuição da amostra. Desse modo, a análise estatística dos resultados deve ser realizada por meio de testes não paramétricos. Por isso, recorreu-se ao teste de Friedman por este ser um teste não paramétrico e permitir avaliar o significado estatístico das diferenças entre as médias obtidas. Os resultados obtidos, indicam que existem diferenças estatisticamente significativas $(p<0,01)$ nas quatro dimensões avaliadas, ou seja, na opinião do profissional de contabilidade, após a implementação do ERP as quatro capacidades/competências analisadas tornaram-se significativamente mais importantes.

Importa analisar os resultados obtidos em cada dimensão separadamente (Quadro 3). Dessa forma, no que concerne à dimensão capacidades/competências em tecnologias de informação, verifica-se que os inquiridos consideram que a importância destas para o desempenho da sua função se alterou com a introdução do sistema ERP $(p=0,000)$. Os dados permitem ainda concluir que o profissional de contabilidade já tinha a percepção de que a necessidade de capacidades/competências em tecnologias de informação era importante $(M=3,54)$. Contudo, após a implementação do sistema ERP tornou-se claro que o profissional de contabilidade está ciente que necessita de significativamente mais capacidades/competências em tecnologias de informação $(M=4,18)$.

Observa-se também, que as diferenças ao nível do conhecimento de outras áreas funcionais da organização antes e após a implementação do ERP, são estatisticamente significativas $(p=0,000)$. O profissional de contabilidade também tem a percepção da necessidade de possuir significativamente mais conhecimentos das outras áreas funcionais da organização após a implementação do ERP (Antes M=3,74; Após M=4,28).

No que diz respeito ao trabalho de equipa com outras áreas funcionais da organização antes e após a implementação do ERP, também aqui as diferenças são estatisticamente significativas $(p=0,000)$. Denotando-se, que o profissional de contabilidade tem a percepção de que a importância do trabalho de equipa com outras áreas funcionais da organização é agora significativamente maior após a implementação do ERP $(M=4,31)$, comparativamente ao que ocorria antes da sua implementação $(M=3,72)$. 
Quadro 3 - Teste de Friedman para as capacidades/competências requeridas antes e após a implementação do sistema ERP.

\begin{tabular}{|c|c|c|c|c|c|}
\hline $\begin{array}{l}\text { Variável } \\
(N=65)\end{array}$ & Grupo & $\begin{array}{l}\text { Proporção } \\
\text { observada }\end{array}$ & Média & Chi-Square & Sig. \\
\hline $\begin{array}{l}\text { Capacidades/Competências } \\
\text { em tecnologias de } \\
\text { informação }\end{array}$ & $\begin{array}{l}1 \text { (Antes da } \\
\text { Implementaçã } \\
\text { o) } \\
2 \text { (Após a } \\
\text { Implementaçã } \\
\text { o) }\end{array}$ & $\begin{array}{l}1,26 \\
1,74\end{array}$ & 3,54 & 29,121 & $0,000 * *$ \\
\hline $\begin{array}{l}\text { Conhecimentos de } \\
\text { áreas funcionais } \\
\text { organização da }\end{array}$ & $\begin{array}{l}1 \text { (Antes da } \\
\text { Implementaçã } \\
\text { o) } \\
2 \text { (Após a } \\
\text { Implementaçã } \\
\text { o) }\end{array}$ & $\begin{array}{l}1,30 \\
1,70\end{array}$ & $\begin{array}{l}3,74 \\
4,28\end{array}$ & 24,143 & $0,000 * *$ \\
\hline $\begin{array}{l}\text { Trabalho de equipa com } \\
\text { outras áreas funcionais da } \\
\text { organização }\end{array}$ & $\begin{array}{l}1 \text { (Antes da } \\
\text { Implementaçã } \\
\text { o) } \\
2 \text { (Após a } \\
\text { Implementaçã } \\
\text { o) }\end{array}$ & $\begin{array}{l}1,27 \\
1,73\end{array}$ & $\begin{array}{l}3,72 \\
4,31\end{array}$ & 30,000 & $0,000 * *$ \\
\hline $\begin{array}{l}\text { Compreensão de ambas as } \\
\text { contabilidades, de gestão e } \\
\text { financeira }\end{array}$ & $\begin{array}{l}1 \text { (Antes da } \\
\text { Implementaçã } \\
\text { o) } \\
2 \text { (Após a } \\
\text { Implementaçã } \\
\text { o) }\end{array}$ & $\begin{array}{l}1,35 \\
1,65\end{array}$ & $\begin{array}{l}3,97 \\
4,34\end{array}$ & 19,000 & $0,000 * *$ \\
\hline
\end{tabular}

** Estatisticamente significativo para um nível de significância de $1 \%$

Fonte: Elaborado pelos autores (2017).

Por último, as diferenças ao nível da compreensão de ambas as contabilidades de gestão e financeira, antes e após a implementação do ERP, são aqui também identificadas estatisticamente significativas $(p=0,000)$, pois se observa que há por parte do profissional de contabilidade uma clara perceção da necessidade de uma maior compreensão de ambas as contabilidades, de gestão e financeira após a implementação do ERP (Antes da implementação do sistema ERP M=3,97; Após a implementação do sistema ERP M=4,34).

Finalmente, foi solicitado aos inquiridos que se pronunciassem sobre o tempo despendido no desempenho de um conjunto de tarefas (referidas no Quadro 4), antes e após a implementação do sistema ERP.

Quadro 4 - Tempo que o contabilista de gestão despende no desempenho das suas funções após a implementação do sistema ERP 


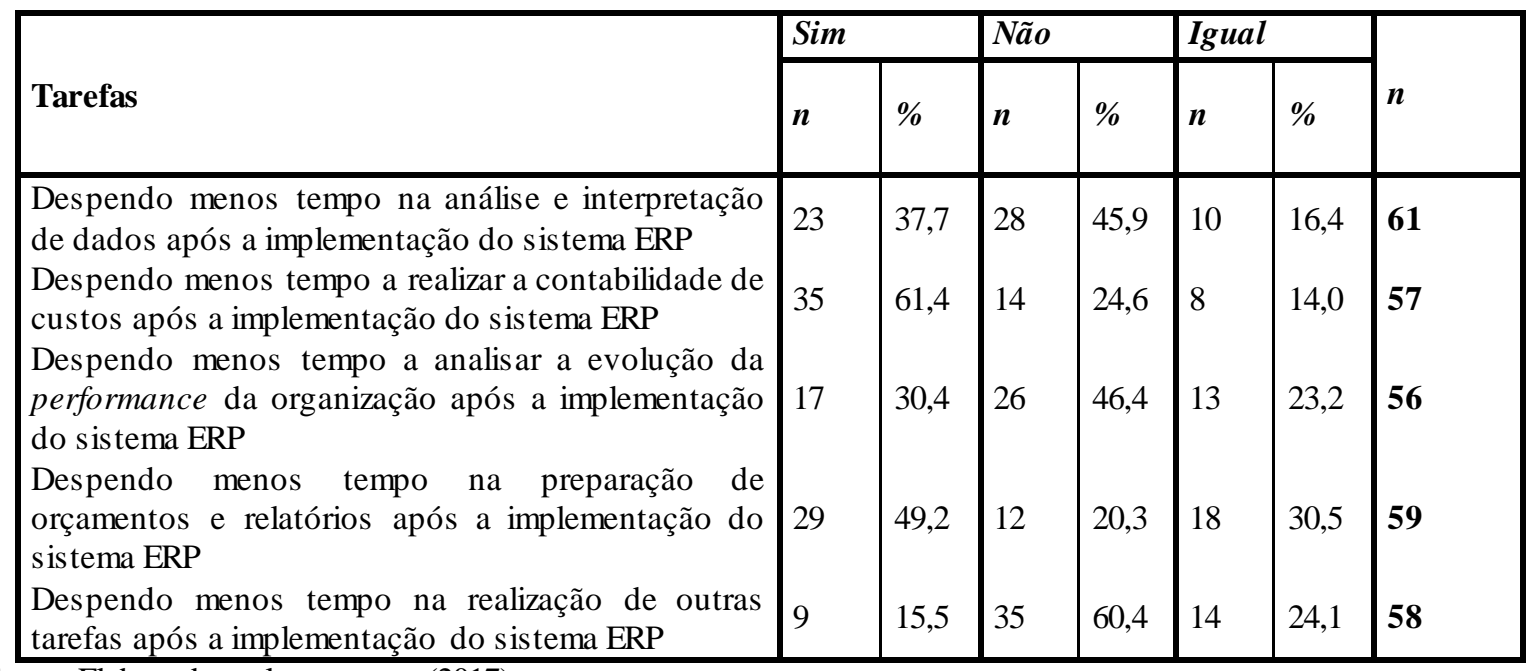

Fonte: Elaborado pelos autores (2017).

Assim, no que se refere, ao tempo que o profissional de contabilidade necessita para realizar suas tarefas, pode-se observar (Quadro 4), que após a implementação do sistema ERP, a maioria dos profissionais de contabilidade relatam necessitar de menos tempo para realizar a contabilidade de custos $(n=35 ; 61,4 \%)$ e preparar os orçamentos e os relatórios $(n=29$; 49,2\%). Em contrapartida, o profissional de contabilidade passa a despender mais tempo para a análise e interpretação de dados $(n=28 ; 45,9 \%)$; para a análise da evolução da performance da organização $(n=26 ; 46,4 \%)$ e para realizar outras tarefas $(n=35 ; 60,4 \%)$.

\section{DISCUSSÃO DOS RESULTADOS}

Nesta investigação, para estudar o impacto do sistema ERP, no papel e nas tarefas do contabilista de gestão, foram formuladas duas hipóteses. Assim, a hipótese 1 sugere que as capacidades/competências requeridas ao contabilista de gestão aumentaram após a implementação do sistema ERP.

Vários estudos (Caglio, 2003; Granlund \& Malmi, 2002; Scapens \& Jazayeri, 2003; Spathis \& Constantinides, 2004) investigaram as mudanças no papel e nas tarefas do contabilista de gestão após a implementação do sistema ERP, destacando o papel do profissional de contabilidade enquanto elemento-chave nas fases de implementação e manutenção dos sistemas ERP (Jean-Baptiste, 2009). Constata-se ainda na literatura, que após a implementação do sistema ERP os contabilistas de gestão revelam mais conhecimentos dos processos de negócio e tornaram-se mais cientes dos objetivos da empresa (Fahy \& Linch, 1999). E, que a implementação do sistema ERP exige ao contabilista de gestão mais 
competências e conhecimentos em tecnologias de informação. No seu estudo Jean-Baptiste (2009) confirma, mesmo, que os profissionais de contabilidade têm maiores hipóteses de integrarem a equipa de implementação do sistema ERP se possuírem bons conhecimentos de tecnologias de informação em paralelo com a sua especialização financeira. Assim, o contabilista de gestão nos dias de hoje necessita de manter atualizadas as suas capacidades/competências profissionais para além de possuir bons conhecimentos em tecnologias de informação, se quiser garantir um bom desempenho neste novo ambiente de trabalho (Maccarrone, 2000; Sayed, 2006; Spathis \& Constantinides, 2004; Spathis 2006).

O estudo realizado por Sayed (2006) permitiu concluir que com a implementação do sistema ERP os contabilistas de gestão passam a ter um maior impacto na definição das necessidades do negócio da organização. Por outro lado, as novas tecnologias de informação permitem que a informação seja facilmente transferível para os "não-contabilistas", como é o caso dos gestores, sendo vistas como um instrumento essencial para a divulgação do conhecimento por toda a organização. Desta forma, o profissional de contabilidade corre o risco de perder o controlo se não desenvolver as competências necessárias, ao nível da comunicação da informação; do trabalho em equipa; do conhecimento do negócio; do pensamento estratégico; bem como capacidades analíticas e interpretativas, de forma a poder vencer neste novo ambiente organizacional. Também Caglio (2003) considera que o contabilista de gestão passou a ser um profissional que simultaneamente necessita de possuir conhecimentos contabilísticos e um profundo conhecimento do negócio.

Os resultados recolhidos nesta investigação, indicam que o profissional de contabilidade após a implementação do sistema ERP, atribui uma maior importância às capacidades/competências em tecnologias de informação; conhecimentos de outras áreas funcionais da organização; trabalho de equipa com outras áreas funcionais da organização e compreensão de ambas as contabilidades, de gestão e financeira. Assim, os resultados apontam para que o profissional de contabilidade tenha de desenvolver as suas capacidades/competências para poder enfrentar os novos desafios colocados pelo novo sistema. Por outro lado, constata-se que as diferenças verificadas ao nível da importância das capacidades/competências, são todas elas estatisticamente significativas ( $p<0,01$ ), o que demonstra que estas capacidades/competências passaram a ser significativamente mais importantes para o profissional de contabilidade após a implementação do sistema ERP.

Desta forma, os dados recolhidos permitem confirmar a hipótese em análise, uma vez que os inquiridos têm uma clara perceção do aumento da importância destas capacidades/competências após a implementação do sistema ERP. Este resultado não 
surpreende e corrobora os resultados obtidos por outros estudos (Caglio, 2003; Fahy \& Linch, 1999; Maccarrone, 2000; Sayed, 2006; Spathis \& Constantinides, 2004; Spathis, 2006).

A Hipótese 2 sugere que o tempo necessário para o contabilista de gestão realizar as suas tarefas mudou após a implementação do sistema ERP.

Granlund e Malmi (2002) alegam que o sistema ERP fornece aos contabilistas de gestão mais tempo para prepararem análises mais sofisticadas. Também Fahy e Linch (1999) constatam que o sistema ERP permite uma redução da carga de trabalho do contabilista de gestão, nomeadamente ao nível da diminuição do tempo necessário para preparar relatórios, libertando o tempo necessário para a realização de análises mais detalhadas.

Com a diminuição do tempo necessário para realizar as tarefas de rotina, Scapens e Jazayeri (2003) referem que, após a implementação do sistema ERP, os contabilistas de gestão se transformaram em verdadeiros analistas. Eles deixam de centrar as suas preocupações na produção de relatórios sobre a performance do negócio, para se focarem mais na interpretação dos indicadores e das suas implicações. Também Caglio (2003) considera que o contabilista de gestão despende menos tempo nas tarefas de relato, porque a maioria destas tarefas estão automatizadas. Isto, permite-lhe dispor de mais tempo para se concentrar na análise do negócio. Esta ideia não é nova, já na década de 90, Lebas alegava que o profissional de contabilidade do futuro estaria envolvido em todas as fases do processo de tomada de decisão, para além das suas funções tradicionais teria de desenvolver um papel de analista e, ao lado dos gestores, tornar se um criador de sistemas e processos, um comunicador e um importante ator na gestão da mudança (Lebas, 1995).

Nas organizações inquiridas, os resultados recolhidos, indicam que o tempo necessário para a realização das tarefas de contabilidade de gestão mudou com a implementação do sistema ERP. Deste modo, o profissional de contabilidade necessita de menos tempo para realizar tarefas tradicionais como a contabilidade de custos e a preparação de orçamentos e relatórios. Sendo, o tempo extra que o profissional de contabilidade passa a ter, utilizado para analisar e interpretar dados, analisar a evolução da performance da organização, ou despendido em outras tarefas da organização. Assim, os dados recolhidos permitem confirmar que após a implementação do sistema ERP se verificou uma mudança quer no tempo necessário para o contabilista de gestão realizar as suas tarefas, quer na natureza das tarefas a desempenhar. Aqui também os resultados recolhidos vêm corroborar os obtidos em estudos realizados em outros países (Caglio, 2003; Fahy \& Linch, 1999; Granlund \& Malmi, 2002; Scapens \& Jazayeri, 2003). 


\section{CONSIDERAÇÕES FINAIS}

Neste estudo, pretendeu-se averiguar qual o impacto do sistema ERP no papel e nas tarefas do contabilista de gestão. Neste sentido, foi estudado o impacto do sistema ERP nas capacidades/competências requeridas ao contabilista de gestão e o tempo necessário ao contabilista de gestão para realizar suas tarefas na organização.

Relativamente, às capacidades/competências requeridas após a implementação do sistema ERP, os resultados recolhidos indicam que o profissional de contabilidade passou a atribuir maior importância às capacidades/competências em tecnologias de informação; aos conhecimentos de outras áreas funcionais da organização; ao trabalho em equipa com outras áreas funcionais da organização e à compreensão de ambas as contabilidades, de gestão e financeira. Pode-se assim concluir, que o profissional de contabilidade tem de desenvolver as suas capacidades/competências para poder responder aos novos desafios colocados pelo sistema ERP.

No que respeita ao tempo necessário para o contabilista de gestão realizar as suas tarefas na organização, os resultados recolhidos indicam que esse tempo mudou com a implementação do sistema ERP. Assim, o profissional de contabilidade passa a necessitar de menos tempo para realizar as tarefas tradicionais como a contabilidade de custos e a preparação de orçamentos e relatórios, podendo dedicar-se a novas tarefas como analisar e interpretar dados, analisar a evolução da performance da organização, bem como realizar outras tarefas na organização.

Em síntese, esta investigação permitiu constatar que a implementação do sistema ERP nas organizações vem exigir ao contabilista de gestão maiores competências e conhecimentos. Assim, podemos concluir que o contabilista de gestão nos dias de hoje para além de possuir bons conhecimentos em tecnologias de informação, necessita de desenvolver proactivamente as suas capacidades nessa área, de forma a conseguir desempenhar seu papel dentro deste novo ambiente de trabalho tecnologicamente mais sofisticado. Por outro lado, tem de desenvolver as competências necessárias: ao nível da comunicação da informação; do trabalho em equipa; do conhecimento do negócio; do pensamento estratégico; bem como capacidades analíticas e interpretativas. Por último, pode-se concluir que após a implementação do sistema ERP se verificou uma mudança na gestão do tempo e na natureza das tarefas exigidas ao contabilista de gestão. 
Finalmente, os resultados deste estudo apenas se podem generalizar para uma população com as características da que foi estudada - empresas portuguesas que implementaram com sucesso o sistema ERP da SAP. A replicação deste estudo em populações com características diferentes pode ser explorada em investigações futuras.

\section{REFERÊNCIAS}

Abdinnour, S. \& Saeed K. (2015). User perceptions towards an ERP system: Comparing the post-implementation phase to the pre-implementation phase. Journal of Enterprise Information Management. 28 (2): 243 - 259.

Aernoudts, R; Boom, T; Vosselman, E \& Pijl, G. (2005). Management Accounting Change and ERP, an Assessment of Research. Recuperado em 6 maio, 2016, de http://ssrn.com/abstract=984201.

Alves, M. C. (2003). Decisores e informação contabilística - sua influência nas decisões empresariais. Tese de Doutoramento, Universidade da Beira Interior, Covilhã.

Alves, M. C. \& Matos, S. I. A. (2013). ERP adoption by public and private organizations - A comparative analysis of successful implementations. Journal of Business Economics and Management. 14(3): 500-519.

Avital, M. \& Vandenbosch, B. (2000). SAP implementation at Metalica: an organizational drama in two acts. Journal of Information Technology. 15(3):183-194.

Berry, A. J.; Coad, A.F.; Harris, E.P.; Otley, D.T. \& Stringer, C. (2009). Emerging themes in management control: A review of recent literature. The British Accounting Review. 41(1):2-20. 
Booth, P.; Matolcsy, Z. \& Wieder, B. (2000). Integrated Information Systems (ERP-Systems) and Accounting Practice - The australian experience. 3a European Conference on Accounting Information Systems (Ecais'2000), Munique, Alemanha, 27-28 de março.

Burns, J. \& Baldvinsdottir, G. (2005). An institutional perspective of accountants' new roles the interplay of contradictions and praxis. European Accounting Review. 14(4): 725757.

Caglio, A. (2003). Enterprise Resource Planning systems and accountants: towards hybridization? European Accounting Review. 12(1):123-153.

Carnegie, G. \& Napier, C. (2010). Traditional Accountants and business professionals: portraying the accounting profession after Enron. Accounting Organizations and Society. 35(3):360-376.

Davenport, T. (1998). Putting the Enterprise into the Enterprise System. Harvard Business Review. 76(4):121-131

Dechow, N. \& Mouritsen, J. (2005). Enterprise resource planning systems, management control and the quest for integration. Accounting Organizations and Society. 30(7-8): 691-733.

Fahy, M. \& Lynch, R. (1999). Enterprise Resource Planning (ERP) systems and strategic management accounting. XXII Congresso Anual da European Accounting Association, Bordéus, França, 5 - 7 de maio.

Ferreira, L. \& Sarmento, M. (2009). Da investigação quantitativa em contabilidade: investigação por inquérito. In Major, Maria e Vieira, Rui (Ed.), Contabilidade $e$ 
controlo de gestão: teoria, metodologia e prática. Lisboa: Escolar Editora, pp. 167208.

Grabski, S.V., Leech, S.A. \& Schmidt, P.J. (2011). A review of ERP research: a future agenda for accounting information systems. Journal of Information Systems. 25(1): 37-78.

Ganassali, S. (2008). The influence of the design of web survey questionnaires on the quality of responses. Survey Research Methods. 2(1): 21-32.

Granlund, M. \& Malmi, T. (2002). Moderate impact of ERPS on management accounting: a lag or permanent outcome?’. Management Accounting Research. 13(3):299-321.

Hyvönen, T. (2003). Management accounting and information systems: ERP versus BoB. European Accounting Review. 12(1):155-173.

Jean-Baptiste, R. (2009). Can accountants bring a positive contribution to ERP implementation? International Management Review. 5(2): 81-109.

Lambert C. \& Sponem, S. (2012). Roles, authority and involvement of the management accounting function: a multiple case-study perspective. European Accounting Review, 21(3), 565-589.

Lebas, M. (1995). Comptabilité de gestion: les défis de la prochaine décennie. Revue Française de Comptabilité. 265, março, pp. 35-48.

Lodh, S. \& Gaffikin, M. (2003). Implementation of an integrated accounting and cost management system using the SAP system: a field study. European Accounting Review. 12(1):85-121. 
Maccarrone, P. (2000). The impact of ERPs on management accounting and control systems and the changing role of controllers. $23^{\circ}$ Annual Congress of the European Accounting Association, Munique, Alemanha, 29 - 31 de março.

Newman, M. \& Westrup, C. (2005). Making ERPs work: accountants and the introduction of ERP systems. European Journal of Information Systems. 14(3): 258-272.

Quattrone, P. \& Hopper, T. (2005). A time-space odyssey: management control systems in two multinational organisations. Accounting, Organizations and Society. 30(7-8):735764.

Quattrone, P. \& Hopper, T. (2001). What does organizational change mean? Speculations on a taken for granted category. Management Accounting Research. 12(4): 403-435.

Ribeiro, J. \& Oliveira, J. (2009). Sistemas Enterprise Resource Planning. In Major \&Vieira (Ed.), Contabilidade e controlo de gestão: teoria, metodologia e prática. Lisboa: Escolar Editora, pp. 335-360.

Rom, A. \& Rohde, C. (2006). Enterprise resource planning systems, strategic enterprise management systems and management accounting - A Danish study. Journal of Enterprise Information Management. 19(1):50-66.

Ruivo, P., Oliveira, T., \& Neto, M. (2014). Examine ERP post-implementation stages of use and value: empirical evidence from portuguese smes. International Journal of Accounting Information Systems, 15(2), 166-184.

Sayed, H. (2006), ERPs and accountants' expertise: the construction of relevance.”, Journal of Enterprise Information Management. 19(1): 83-96. 
Scapens, R. \& Jazayeri, M. (2003). ERP systems and management accounting change: opportunities or impacts? A research note. European Accounting Review. 12(1): 201233.

Scapens, R.; Jazayeri, M. \& Scapens, J. (1998). SAP: integrated information systems and the implications for management accountants. Management Accounting: Magazine for Chartered Management Accountants. 76(8):46-48.

Shang, S. \& Seddon, P. (2002). Assessing and managing the benefits of enterprise systems: the business manager's perspective. Information Systems Journal. 12(4):271-299.

Simons, P. (2012). Management accountants must learn to speak in the language of the business to be better business partners. Controlling and Management. 56(1): 39-43.

Spathis, C. (2006). Enterprise systems implementation and accounting benefits. Journal of Enterprise Information Management. 19(1): 67-82.

Spathis, C. \& Constantinides, S. (2004). Enterprise Resource Planning Systems' impact on accounting processes. Business Process Management Journal. 10(2): 234-247.

Vanti, A.; Ferreira, L.; Janikian, A. \& Ferro, C. (2003). O novo perfil do profissional de contabilidade frente às inovações da tecnologia da informação (TI) como em casos de implantação de sistemas integrados de gestão. Revista de Administração $e$ Contabilidade. 1(2): 89-116.

Wu, J.; Shin, S. \& Heng, M. (2007). A methodology for ERP misfit analysis. Information \& Management. 44(8): 666-680. 